Virology

\title{
Human leukaemia virus mimics cellular antigens
}

\section{from Robin A. Weiss}

THE human T-cell leukaemia virus (HTLV) is yielding intriguing data on unexpected relationships between viral and host antigens that have not previously been seen with animal retroviruses. Earlier this year Haynes et al. ${ }^{1}$ showed that a monoclonal antibody against the p19 gag antigen of HTLV cross-reacts with an epithelial antigen in the human thymus. As HTLV is not an endogenous virus ${ }^{2}$ one would not have expected to find a related antigen expressed in normal tissue. Although incidental cross-reactions between otherwise unrelated proteins are increasingly noted with monoclonal antibodies, the thymus antigen has the same molecular weight as the viral antigen ${ }^{3}$ and the relationship between the two antigens seems more than chance.

It can be argued that viral p19 is not, after all, a virally coded antigen, but is a cellular antigen induced in HTLVtransformed thymus-derived cells, and that antigen becomes associated with viral core protein during assembly. This seems unlikely because the monoclonal anti-p19 antibody also recognizes a precursor protein containing both p19 and p24 antigens; p24 is indisputably a gag gene product and it is not expressed in the normal thymus. With the sequence of the HTLV genome known ${ }^{4}$, it should be possible to establish by partially sequencing the p19 protein whether it really is encoded by the gag gene.

Now new evidence reported in this issue of Nature suggests the envelope glycoprotein of HTLV is also related to cellular antigens. Mann et al. ${ }^{5}$ (see p.58) from the $\mathrm{Na}$ tional Cancer Institute, Uniformed Services University of the Health Sciences and Duke University School of Medicine report that cell lines producing HTLV express supernumerary HLA specificities, which are determined by the virus. Using alloantisera and a monoclonal antibody to a set of HLA class I antigens, all HTLVtransformed cell lines are found to express an antigen not always found in uninfected cells of the same host. The new HLA specificity could be a cellular antigen induced by HTLV, but more probably it is a determinant of the viral envelope antigen. In hybrids of HTLV-infected HUT102 cells and hamster cells, the new HLA specificity segregates not with HLA on chromosome 6 , but with the chromosome carrying the integrated HTLV provirus ${ }^{6}$.

In an accompanying paper, Clarke et al. ${ }^{7}$ (see p.60) from the National Cancer Institute show that a weak but real homology of nucleotide sequences exists between DNA clones of the env gene of HTLV and class I HLA. This finding makes the an- tigenic cross-reactivity much more significant. The homologous sequences are in the coding region for the extracellular portion of these membrane antigens. It will be interesting to compare the full nucleotide sequences of HTLV env and the homologous region of $H L A-B$.

It is not yet clear what the biological significance might be for a viral membrane antigen to mimic a histocompatibility antigen. Mann et al. ${ }^{5}$ suggest that it might render the leukaemia patients tolerant to HTLV membrane antigens. However, our studies with HTLV pseudotypes indicate that all patients with adult $\mathrm{T}$-cell leukaemia make neutralizing antibodies specific to HTLV envelope glycoprotein ${ }^{8}$ as well as antibodies to viral core proteins. It will be interesting to investigate whether individuals with the HLA haplotype to which the viral antigen is related are less or more susceptible to HTLV infection and HTLVassociated malignancy.

Yet another cellular antigen associated with the HTLV envelope is the T-cell growth factor receptor recognized by the tac-1 monoclonal antibody 9 . In this case, however, it is clear that the antigen is cellular in origin and is selectively taken up from the cell membrane during budding of the virions ${ }^{10}$.

Robin A. Weiss is at the Institute of Cancer Research, Fulham Road, London SW3 $6 J B$.

. Haynes, B.F. et al. J. exp. Med. 157, 907 (1983).

2. Reitz, M.S., Poieš, B.J., Ruscetti, F.W. \& Gallo, R.C. Proc. natn. Acad. Sci. U.S.A. 78, 1887 (I981)

Gallo, R.C. Personal communication.

4. Seiki, M., Hattori, S., Hirayama, Y. \& Yoshida, M. Proc. natn. Acad. Sci. U.S.A. 80, 3618 (1983)

5. Mann, D.I. et al. Nature 304, 58 (1983).

6. Reit<, M. \& O'Brien, S. Personal communication.

7. Clarke, M.F., Gelmann, E.P. \& Reitz, M.S. Nature 304,60 (1983).

8. Clapham, P., Nagy, K. \& Weiss, R.A. Unpublished data. 9. Leonard, W. J. et al. Nature 300, 267 (1983)

10. Lando, Z. et al. Nature (in the press).

\section{Stereochemistry}

\section{Handedness from symmetry and a stereochemical coupe du roi}

\section{from Stephen Mason}

STIMULATED by an after-dinner diversion known as la coupe du roi, Anet et al. ${ }^{1}$ have contrived a stereochemical feat that at first sight challenges a general principle originally enunciated by Louis Pasteur.

Pasteur $^{2}$ was the first to identify the class of handed or enantiomorphous molecules, which are characterized by structures that are not superposable by translation and rotation alone upon the corresponding mirror-image form. Such structures were termed 'dissymmetric' by Pasteur ${ }^{2}$ and, subsequently, 'chiral' by Kelvin ${ }^{3}$ from the

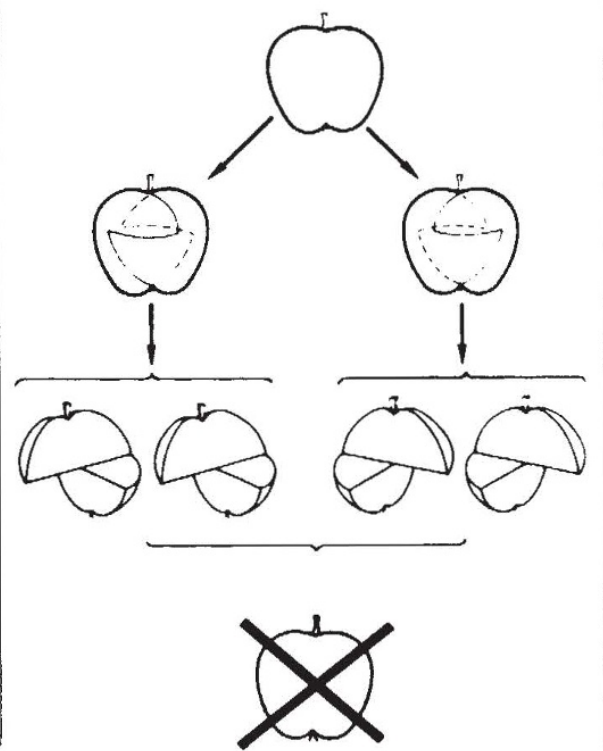

familiar analogy of the morphological relationship between the left and the right hand.

Fischer ${ }^{4}$ introduced a convention to distinguish between the two mirror-image molecular forms, one being termed the D-isomer (dextro) and the other the L-enantiomer (laevo). Although still used for the $\alpha$-amino acids and the sugars, the Fischer convention accummulated ambiguities of nomenclature and was replaced. In the generally adopted system of Cahn, Ingold and Prelog 5 , based on the relative mass of the atoms bonded to an asymmetric carbon atom or other chiral centre, the absolute structure of a given chiral molecule is unambiguously specified as that of the $R$ configuration (rectus) or the $S$ configuration (sinister).

Pasteur ${ }^{2}$ maintained that, in the laboratory, the chemist without dissymmetric agents at his disposal is able to synthesize only an equimolecular mixture of $R$ and $S$ isomers, a racemic mixture, or an internally compensated single product, with bonded $R$ and $S$ moieties, the so-termed meso-substances. Similarly, the chemical degrada-

Fig. 1 The apple subject to la coupe du roi. Each apple yields a pair of homochiral segments, the two being identical in the ideal case of the sphere. The particular handedness of the two segments depends on the pair of opposite equatorial quadrants chosen for the horizontal cuts. A left-handed segment, given by one of the choices, and a right-handed segment, provided by the other, cannot be reassembled to form an apple. 